Analyse stylistique de l'écriture de Georges Simenon dans La Vérité

\title{
Analyse stylistique de l'écriture de Georges Simenon dans La Vérité
} sur Bébé Donge

Par

Wael Fouad Naguib

Maitre de conférences

Faculté de Pédagogie, Université d'Ain Chams

«On commence à écrire de la fiction, on commence à raconter des histoires, pour essayer d'atteindre ce qui est de la vérité de l'existence humaine.» ${ }^{(1)}$

Analyse stylitique de l'écriture de Geroges Simenon dans La vérité sur Bébé Donge Presenté par Dr Wael Fouad Naguib Ibrahim, Maitre de conférences au departement de la langue française et ses Lettres, à la Faculté de Pédagogie , l'Université d'Ain Chams. (01228506421 wattsapp) L'article commence par une brève introduction sur la justification du choix de l'auteur et de l'oeuvre étudiée tout en mettant en relief la pensée du romancier. Ensuite, nous avons procédé à déterminer les traits caractéristiques de l'oeuvre faisant le corps de la recherche pour la classer en s'appuyant sur le titre de l'oeuvre. Ce point nous a amené à étudier l'organisation du récit et préciser par qui la narration a été faite tout le long du récit, la fréquence des événements.Nous avons du développer les différentes fonctions du narrateur principal sans oublier de braquer la lumière sur l'étude des personnages, l'ordre de leur apparition, et l'objectif de cet ordre. Naturellment, le schéma actantiel a été l'étape suivante de l'analayse de l'écriture de Simenon dans son roman en question. En fait, il a été important d'analyser l'emploi fréquent de certains temps verbaux qui ont dominé le récit. L'analyse de deux figures de style (la comparaison et la répétition) a précédé la conclusion et la bibliographie .

${ }^{(1)}$ FALLOIS, Bernard de : Simenon, Paris, Gallimard, 1985, p. 250. 


\section{G. Simenon}

Dr Wael Fouad Naguib

Le but de notre recherche est de mettre en relief l'originalité de l'écriture de Georges Simenon dans La vérité sur Bébé Donge. Parmi les raisons du choix de cette œuvre, c'est qu'elle a été mise sur le petit écran par de très célèbres acteurs. C'est l'un des romans de l'un des romanciers les plus traduits dans le monde entier. Simenon est un auteur d'origine belge et il choisit le cadre socio-politique après la première année de la deuxième guerre mondiale 1939-1945 pour aborder un sujet important, le problème de l'existence et les premières analyses de la philosophie de l'existentialisme, en vigueur en ce temps-là. L'œuvre a été imprimée à «Vouvant, le 7septembre 1940» ${ }^{(2)}$.

Le même cadre socio-politique démontre la position de Simenon qui a décidé de ne pas rejoindre l'armée, de se déconnecter du monde qui l'entourait et de ses problèmes, de choisir de mener sa propre existence indépendamment des souffrances de la race humaine. L'un des meilleurs spécialistes de Simenon, Michel Carly, dans Simenon, les années secrètes : 1940-1945, d'après son enquête et les témoignages recueillis - affirme "que Simenon n'a pas été un " collabo », mais que, comme beaucoup à cette époque, il a été un peu lâche (il n'est pas revenu en Belgique, afin d'échapper au service militaire), un peu rusé et opportuniste, sans aucun sens de l'histoire avec un grand $H{ }^{(3)}$. La personnalité et les idées de Simenon avant 1940 n'étaient plus celles d'après.

Presque toujours, Simenon démontre que le sujet principal de sa production littéraire est l'homme qui " est le même partout » ${ }^{(4)}$ et qu'il désigne, faute d'avoir trouvé meilleure définition par «l'homme $n u »{ }^{(5)}$ Tout le long du roman, le terme "existence"en tant qu'équivalent de la condition humaine a été répété à maintes reprises et à titre d'exemple on cite:«Du moment qu'elle (Bébé Donge)ne se mêlait pas de lui compliquer l'existence........» ${ }^{(6)}$ François croyait que

\footnotetext{
(2) SIMENON, Georges : La Vérité sur Bébé Donge, Paris, Gallimard, 1940, p.246

(3) CARLY, Michel : Simenon, les années secrètes : Vendée 1940-1945, Le Châteaud'Olonne : Éd. d'Orbestier, 2005, 180 P. WC,

${ }^{(4)}$ LEURRRIN, Michel et SCHWERIGHAEUSER, Jean Paul : Le Guide du polar, Histoire du roman policier français, Paris, Syros, "Les Guides culturels", 1987, p.49.

${ }^{(5)}$ DE FALLOIS, Bernard : Simenon, Paris, Gallimard, coll. "Bibliothèque Idéale",1961, p.36.

${ }^{(6)}$ SIMENON, Georges: op.cit. p.15. 
Analyse stylistique de l'écriture de Georges Simenon dans La Vérité son épouseBébé se plaisait de rendre la vie insupportable. Le concept de "l'existence ${ }^{(7)}$ a toujours étéau centre d'intérêt de Simenon.

Dans Ecrire l'homme, M. LEMOINE résume que l'œuvre de G. SIMENON «doit-être perçue avant tout comme une recherche de la condition humaine. En fait, Bébé s'est prononcée d'attenter à la vie de son mari non pour se marier avec une autre personne qu'elle aime ou qui l'aime, non par "jalousie" ${ }^{(8)}$ «mais parce qu'elle se meurtrissait à son indifférence.» ${ }^{(9)} \mathrm{La}$ vérité sur Bébé Donge est un exemple type de roman racontant la souffrance de la solitude au sein du mariage et même au sein de la famille. C'est un drame de l'existence humaine vide.

En tant que "roman à thèse" ${ }^{(10)}$, le roman renforce l'idée que ni l'opulence, ni le mariage n'assurent ni le bonheur humain ni l'entente du couple ni l'évasion à la solitude humaine. La preuve en est la vie accablante des jeunes mariés Bébé et François avant et après leur mariage. Au cours de la lecture de l'œuvre, de nombreux termes soutenant l'idée de l'accablement, de la déception et de l'incompréhension partagée ont été fréquents comme : «exaspéré, effrayé, affalé, affolé, gêner, sanglots, ennuyé, soupirer, intimider, jalousie, haine, crainte, lassitude, désordre écœurant, les peurs, la rage, la solitude, l'angoisse, les souffrances, la honte, la cruauté morale $\gg{ }^{(1)} \mathrm{C}$ 'est un roman noir.

En plus, Selon M. LEMOINE, «Même dans les romans dits policiers, il importe moins, pour Simenon, de savoir qui a tué que de savoir quel homme est l'assassin, quel homme était la victime et...qui nous sommes ${ }^{(12)}$.La vérité sur Bébé Donge n'est pas un roman policier au sens propre du terme malgré le crime perpétré, l'attentat de Bébé à la vie de François par l'arsenic, la décision de ce dernier de

\footnotetext{
(7) LEMOINE, Michel : Ecrire l'homme, Paris, Gallimard, 2003, p.94.

(8) DE FALLOIS, Bernard : Simenon, Paris, Gallimard, col. "Bibliothèque Idéale" 1961, p.36

(9) SIMENON, Georges : La vérité sur Bébé Donge, Paris, Gallimard, p.88, cf. p.89, 94,124,129,159 159,159, 159, 160, 161, 171, 209, 210.

(10) Le roman à thèse : https://www.larousse.fr/portail/cf.SUZAN,Sully-SULEIMANN,Le roman à thèse, Paris, PUF. 1979, p.22

${ }^{(11)}$ SIMENON, Georges: Op.cit. p. 225, cf.p.16,20,36,37,38,41,42,44,78,81,93,101,107,158,162,190

${ }^{(12)}$ LEMOINE, Michel :loc.cit.
} 
Dr Wael Fouad Naguib

l'acquitter à la fin du roman et l'explication qu'elle a donnée. Après la perpétration du crime, elle a été arrêtée par la police, traduite en justice et condamnée à cinq ans de prison. Elle n'a nié ni le crime, ni les mobiles, ni l'arsenic utilisé. Au début du roman, le lecteur apprend qu'il s'agit «du dimanche $d u$ grand drame.» ${ }^{(13)}$ Quelques pages après, le lecteur est préparé à imaginer le problème de l'héroïne mentionné par l'épicier de la province «...M. Jacques va bien? A la campagne, il doit profiter. Et Mme Donge? Elle ne s'ennuie pas, toute seule ?» ${ }^{(14)}$ alors qu'elle vivait dix ans auprès de son mari François.

En fait, le titre captivant de l'œuvre est intéressant à analyser. La vérité sur Bébé Donge. Nous avons l'impression que Simenon ne prend pas la défense de Bébé seulement mais de la femme en général. Ceci donne plus de poids au titre de l'œuvre qui aurait pu être aussi "Le drame de François", ou "FrançoisVictime" mais il a préféré à tous La Vérité sur Bébé Donge. Le prénom de l'héroïne Bébé ne figure pas seul sur la couverture du roman, mais il est accompagné d'un nom de famille Donge. C'est une manière pour faire travailler l'esprit du lecteur qui va penser au nom de famille Donge et du prénom Bébé. Est-elle la fille de monsieur Donge ou bien c'est le nom de famille du conjoint?.

Le titre fait hésiter le lecteur entre un drame conjugal ou une autobiographie de l'héroïne Bébé. La réponse est claire grâce aux phrases suivantes: «D’autres dimanches, pour les Donge, sont restés ....ou encore le dimanche de la grande dispute, celle qui a brouillé les deux ménages pour plusieurs mois...» ${ }^{(15)}$ Dès le début, la question est tranchée que c'est un drame conjugal. En plus, le romancier a choisi comme événement majeur "granddrame" ${ }^{\prime(16)}$, comme cadre spatial des événements constituant la scène du crime "LaChataigneraie" ${ }^{\prime(17)}$ et pour le cadre temporel «Ce dimanche, au contraire qu'on pourrait appeler le dimanche $d u$ grand drame s'écoula avec la limpidité et le calme d'un ruisseau en plaine.» ${ }^{(18)} \mathrm{A}$ la deuxième page du roman, le romancier a précisé la date de

\footnotetext{
(13) SIMENON, Georges: Op.cit., p.9.

(14) SIMENON, Georges: Op.cit.p.15

(15) Ibid.p.39, cf.p.50,52,53,65,75,109,134,144,149,163,247

(16) Ibid.p.9

${ }^{(17)}$ Idem.

${ }^{(18)}$ Idem.
} 
Analyse stylistique de l'écriture de Georges Simenon dans La Vérité

l'événement marquant qui est le crime "On était le 20 août.» ${ }^{(19)}$ Les actions se déroulent en plein été et à la campagne. Simenon ne fait perdre ni le temps ni l'attention du lecteur en affirmant : "Il y avait deux frères et deux sæeurs dont le sort avait fait deux ménages.» ${ }^{(20)}$ Elle est la première femme mentionnée dans le roman ensuite François. Ce n'est pas donc l'autobiographie de Bébé. Est-elle l'autobiographie fictionnelle inexacte de Simenon lui-même?

John Simenon «Les romans de mon père ne sont pas purement fictionnels, et, sans être autobiographiques, ils sont nourris de sa vie, de ses expériences, de ses observations.» ${ }^{(21)}$ Simenon lui-même a dit au cours de son interview avec Laure Gerbaud, «Si je me suis toujours mis dans la peau de mes personnages, le temps du roman en cours, mes personnages, si je puis dire, ne se sont jamais mis dans la mienne, plus exactement, aucun n'a été mon reflet» ${ }^{(22)}$. "L'état d'esprit d'un génie de l'écriture: Georges Simenon" ${ }^{(23)}$ d'après l'interview de CARRON avec Simenon.

Pour la narration des événements du roman La vérité sur Bébé Donge, les événements sont narrés en neuf chapitres de longueur presque égale clairement indiqués avec des espaces blancs et des chiffres romains. A l'intérieur de chaque chapitre, nous avons remarqué que la narration a été interrompue par "l'astérisque" ${ }^{\text {(24) } q u e}$ le narrateur utilise pour plusieurs raisons comme par exemple pour marquer la fin d'une phase de narration et en "commencer" ${ }^{\text {(25) }}$ une autre dans le même chapitre. Nous analysons un seul exemple.

Avant l'astérisque, dans le deuxième chapitre, nous avons su comment le crime a été perpétré par Bébé et en présence de quels autres personnages, et comment François a été sauvé à la maison. Après l'astérisque, nous passons à une description de la rue, l'arrivée

\footnotetext{
(19) Ibid., p.10.

${ }^{(20)}$ Ibid., p. 18 .

(21) CARRON, Jérome, Interview de John Simenon, Point de vue, 3juillet 2019 (pressreader).

(22) CARRON, Jérome, Interview de John Simenon, Point de vue, 3juillet 2019 (pressreader)

${ }^{(23)}$ GAUBERT, Laure : "L'état d'esprit d'un génie de l'écriture : Georges, Simenon, https : II www. Osez-écrire-votre-roman.com21février 2018.

${ }^{(24)}$ SIMENON, Georges: Op.cit. p.39, Cf. p.50, 52, 53, 65, 75, 109, 134, 144, 149,163, 247.

${ }^{(25)}$ Ibid.p.39
} 
Dr Wael Fouad Naguib

de l'ambulance de la clinique, de longues discussions entre Bébé et sa mère madame d'Onneville, entre Jeanne et Félix, ou entre le docteur Pinaud et Jeanne, Jeanne et Bébé, Bébé et la bonne, Jeanne et sa mère, François et Félix. Toutes ces discussions aident le lecteur à comprendre ce qui est nécessaire pour la suite du roman.

A la fin de cette phase de narration sous forme de dialogues qui donnent plus de vivacité à la narration, nous passons à un autre astérisque qui sépare une narration trop courte concernant l'annonce de l'arrivée de l'inspecteur Janvier. La séquence suivante est un peu plus longue que la précédente. Il s'agit d'une conversation entre Jeanne, le docteur, l'inspecteur, Bébé et Marthe sa femme de chambre pour se préparer à partir avec la police après l'interrogatoire. Un dernier astérisque avant la fin du chapitre pour souligner le début de l'interrogatoire de l'inspecteur avec Bébé. Pour garder le lecteur en haleine, le troisième chapitre n'a pas été commencé par l'interrogatoire de Bébé mais par la narration de ce qui va se passer avec François à l'hôpital.

Nous avons aussi relevé que l'écriture de la narration et les dialogues dans le roman a été faite uniquement sur les papiers sans être prononcés malgré le discours direct. Il y a des mots en italique. Pour plus de clarification, nous citons les exemples suivants extraits $\mathrm{du}$ roman : «...Je suis allé à la Chataigneraie...»" ${ }^{(26)}$. Selon le site Wikipédia, la Chataigneraie «est une commune non polarisée, qui ne fait donc partie d'aucune aire urbaine ni d'aucun espace urbain» ${ }^{(27)}$

Nous pouvons l'expliquer par la volonté du romancier de s'adresser au plus large public en choisissant un cadre spatial rural suffocant pour une fille vivant dès son bas âge dans le plus beau quartier de Constantinople. De même, s'il avait choisi par exemple une ville comme Paris, il ne serait pas arrivé à bien mettre en lumière les souffrances de Bébé. Chaque lecteur masculin peut se trouver dans la peau du sévère François poussant Bébé à l'attentat à la vie de son mari. Le romancier a voulu discuter et soulever un problème important de son époque.

\footnotetext{
${ }^{(26)}$ SIMENON,Georges :op.cit.p.16, cf.17,20,23,33,34,

${ }^{(27)} \mathrm{La}$ Chataigneraie : https://fr.wikipedia.org/wiki/Wikip.Accueil-principal SIMENON, Georges : Op.cit.p.9 cf. p.16,17,23,33,34,47,50,59,60,63,65,68,81,85,91,114,137,139, $141,144,156,160,162,172,188,190,201,203,211,216$ 
Analyse stylistique de l'écriture de Georges Simenon dans La Vérité

A titre d'exemple d'autres mots écrits en italiques, nous citons «Le Lui avait une majuscule. Bébé ne parlait pas d'un homme comme les autres, pas même de son mari. Elle parlait de Lui. Et elle ne Lui en voulait pas de l'avoir accusée.»(28)

En fait, les dernières phrases de Bébé parlant avec François vers la fin du roman quand il a demandé la réconciliation, nous font comprendre que François ne signifiait plus rien à Bébé,il ne lui était qu'un homme sans aucun lien avec elle. Sur le plan affectif, aucun lien n'existe plus entre eux. Ils ne forment plus un seul couple.

Si nous mettons ces mots écrits tout le long du roman en lettres italiques les uns à côté des autres, nous finissons par nous rendre compte que ces mots et phrases font ensemble le résumé du roman dès la première rencontre de Bébé et de François, leur mariage, la décision de faire un enfant, l'indifférence de Bébé à l'égard de la bonne santé sexuelle de François avec les filles de joie, le refus de la demande de Mme Flament, la vision de l'arsenic par Bébé, la décision de tuer François puis le renvoi de l'amante de François madame Flament, l'exécution du drame, l'arrivée de l'inspecteur, la confession du crime par Bébé, le retour en arrière au jour du crime par Bébé, la confession de Bébé d'avoir attenté à la vie de François qui passait son temps aux bars avec les danseuses.

Ensuite, nous découvrons les drogues auxquelles elle s'était habituée au cours de sa jeunesse. Jeanne a affirmé à François que Bébé vivait en pleine solitude. Bébé a été traduite à la Justice. Nous avons ensuite l'avis du Maitre Boniface sur le crime, et la déclaration que François était accusé de cruauté morale envers Bébé. A la fin de cette suite d'événements, nous arrivons à l'acquittement de Bébé par François, la reconnaissance de celle-ci de sa responsabilité de l'avoir poussée à le tuer. Le premier mot en italique c'est le pronom personnel objet indirect "(Lui $)^{\prime(29)}$ qui s'oppose à la fin au pronom personnel objet direct (la) pour marquer la différence totale des deux caractères. Le pronom personnel objet indirect (lui) dépend d'une préposition qui l'attache à un autre nom ou bien d'autres attaches

\footnotetext{
${ }^{(28)}$ Ibid.p.44cf.p.46,50,52,59,69,70,70,71,80,83,86,87,88,89,113,126,132,146,154,156, 180 , $196,205,213,222,229$,

(29) SIMENON, Georges: op.cit. p. 10,cf.p.14,26,
} 
Dr Wael Fouad Naguib

tandis que le pronom personnel objet direct (la) ne dépend pas d'une préposition, il est seul comme Bébé l'est. Elle ne s'attache qu'à sa fonction de mère.

Les lecteurs comprennent le nœud du roman quand ils lisent la longue discussion de Bébé avec sa sœur Jeanne, le procureur, le substitut du Parquet, l'inspecteur. Vers la fin du roman, le lecteur a lu une longue discussion entre l'inspecteur et Bébé, puis de Bébé avec le maitre Boniface qu'elle a choisi pour la défendre. Enfin, ila pu découvrir le vrai mobile du crime : c'est"la cruauté morale $^{\text {(30) }} \mathrm{du}$ mari envers son épouse, comme les Américains l'appellent.

Une question s'impose aux lecteurs pourquoi n'a-t-elle pas accepté ses conditions d'épouse marginalisée de la vie de son mari ? Elle avait besoin d'éprouver le sentiment de "délivrance"(31) éprouvé après l'attentat à la vie de son mari. Une dernière phrase prononcée par Bébé à François justifie le crime, le refus de la réconciliation proposée par François à la fin du roman : «Le respect humain n'existait plus.» ${ }^{(32)}$ Bébé avait toujours reproché à François son regard moqueur. Cette leçon de vie justifie encore une fois le choix du sujet de la recherche, du roman et du romancier. En France, au cours des années où se sont déroulés les événements du roman, la femme française mariée n'avait pas droit au divorce. «Les époux mariés depuis moins de 3 ans ne peuvent pas divorcer» ${ }^{(33)}$. Bébé a proposé à François de se séparer d'elle quand il voulait: "Le désir légitime d'entrer enfin en possession de sa femme... Un vieux fond de tradition....» ${ }^{(34)}$

François poursuivait ses aventures avec les femmes sans accepter de se divorcer, sans changer sa manière avec Bébé, sans essayer de comprendre sa personnalité. Simenon s'est marié une deuxième fois aprèsson désaccord avec sa première femme. De même, Bébé a été obligée de "supprimer" ${ }^{(35)}$. François au lieu de se suicider parce que «Un enfant a davantage besoin d'une mère que d'un

\footnotetext{
${ }^{(30)}$ Ibid., p.225.

${ }^{(31)}$ Ibid., p. 215.

${ }^{(32)}$ Ibid., p.243.

${ }^{(33)}$ www.http :La situation de la femme en France au cours des années 1940,

${ }^{(34)}$ Ibid., p. 243 .

${ }^{(35)}$ Ibid. p.211 
Analyse stylistique de l'écriture de Georges Simenon dans La Vérité

père....» ${ }^{(36)}$ Ceci éclaircit le nœud du roman, explique le choix du crime comme astuce de la narration de la vie du couple à un moment aussi critique.

Quant à l'ordre de la narration, nous l'analysons selon la théorie de G. Genette et nous relevons que le procédé dominant c'est le flash-back soit après le crime, soit après l'arrestation de Bébé puis la narration a accompagné le déroulement du reste des événements jusqu'à la sentence de l'emprisonnement de Bébé. Le narrateur omniscient a eu recours au flash-back pour la narration du passé de certains personnages importants et leurs complexes psychiques. Nous allons avancer quelques exemples du "flash-back"(37) sans oublier qu'il y a des événements du type "singulatif "(38) c'est-à-dire narré pour une seule fois alors que d'autres sont du type "itératif("(39) c'est-à-dire répétés au cours de la narration. Parmi ces derniers nous citons les suivants: la rencontre hebdomadaire chaque dimanche à la Chataigneraie, dans la première page du roman, nous avons relevé la répétition du jour de dimanche : " dimanche-là, ...D'autres dimanches,le dimanche...ou encore le dimanche... D'autres dimanches, pour les Donge, sont restés en quelque sorte historiques, comme le dimanche ...» ${ }^{(40)} 6$. En voici, un autre exemple «C'était tous les dimanches à recommencer! François faisait le marché le samedi,...chacun lui envoyait sa liste...» ${ }^{(41)} \mathrm{La}$ scène majeure c'est la déclaration de François de ne pas vouloir traduire Bébé à la Justice : «...Je ne veux absolument pas, docteur...» ${ }^{(42)}$, la reprise de l'événement de l'attentat à la vie de François par Bébé par le narrateur omniscient puis par le juge:«...Le dimanche 20 aout, vous trouvant dans votre propriété de la Chataigneraie, commune d'Ornaie, vous avez été victime d'une tentative d'empoisonnement.» ${ }^{(43)}$ Laquestion posée par Bébé sur le nombre de morceau de sucre le temps même du

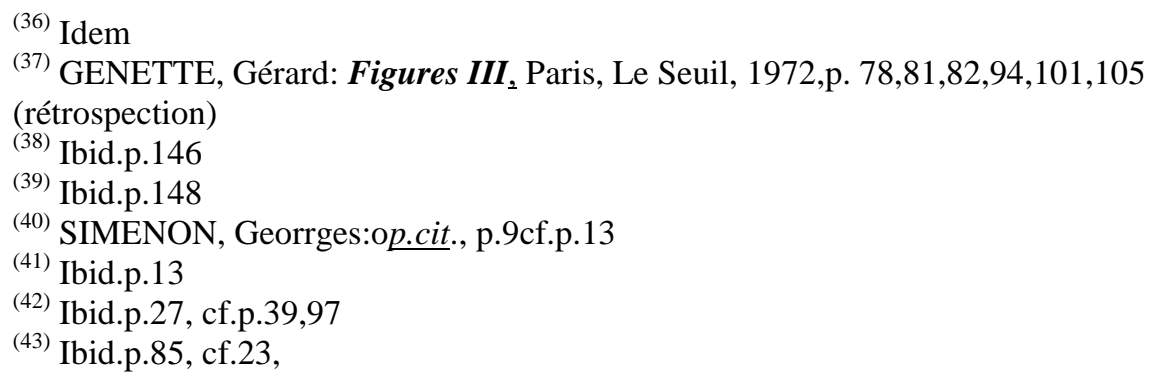


Dr Wael Fouad Naguib

crime et après le crime dit par François: «Un morceau de sucre, maman ?...» ${ }^{(4)}$ Quand François s'est rendu compte du mauvais gout du café, la raison pour laquelle, il a refusé de partager sa constatation pour la même raison, donc le même incident et la même raison ont été cités : «Parce qu'il avait l'habitude de garder ses réflexions. De même, il manquait de chaleur humaine à cause de la ressemblance à sa mère : ...Rien qu'à cause de ce trait, François ne pouvait le renier.» ${ }^{(4)}$ De même, Bébé a répété à François :«Tu as rencontré beaucoup de gens ?» ${ }^{(46)}$ Ajoutons d'autres exemples. La discussion entre Bébé et François sur le plan de faire un enfant tout de suite ou deux trois ans après le mariage : «...Cela t'ennuierait que nous n'ayons un enfant que dans deux ou trois ans ?...

«...Tu es fâché de ce que je t'ai dit l'autre jour ?...»

Pour en arriver, un beau matin, à déclarer comme on traite une affaire

:

«...Je voudrais un enfant tout de suite... » ${ }^{(47)}$

Vers la fin du roman, le narrateur omniscient a narré que François se sentait :«... Idiot! ...Idiot !...Idiot !...» ${ }^{(48)}$ et au cours sa discussion avecJeanne, François a répété qu'il éprouvait le même sentiment de se sentir idiot !

Bébé a répété à plusieurs reprises à François qu'elle aurait voulu con- naitre son père :« ...J'aurais aimé connaitre ton père... Cela n'indiquait-il pas qu'elle, de son côté, avait fait un effort ${ }^{(49)}$ Jeanne a raconté à François le passé de Mme d'Onneville. La narration du même passé a été reprise par Me Boniface : «Nous habitions (à Stamboul) un grand appartement...et il y avait sans cesse des visites...» ${ }^{(50)}$. Enfin, le même narrateur omniscient a rapporté en détails l'interrogatoire fait par le juge à Bébé mais le même interrogatoire a été raconté encore une fois en quelque sorte par le maitre Boniface.

\footnotetext{
${ }^{(44)}$ Ibid.32, cf.p.33

${ }^{(45)}$ Ibid.p.151, cf.p.10,11

(46) Ibid.p.155, cf.p.157,158,

(47) Ibid.p. 154

${ }^{(48)}$ Ibid.p.156, cf.p.166

${ }^{(49)}$ Ibid.p.184, cf. p.122,169

${ }^{(50)}$ Ibid.p.169, cf. p.202
} 
Analyse stylistique de l'écriture de Georges Simenon dans La Vérité

«Je (Bébé) sentais confusément que nous en arriverions là(le crime), mais je n'en étais pas sure. ${ }^{(51)}$ est 1'un des exemples des événements singulatifs les plus illustratifs. Nous n'en avons pas plusieurs illustratifs dans le roman. Après les premiers indices de souffrance de François au momentde boire le café empoisonnant, Bébé «Les trois femmes se regardèrent.» ${ }^{(52)}$ «La porte s'entrouvrit pour laisser passer le docteur mais se referma devant Bébé Donge....» ${ }^{(53)}$ François a refusé à son épouse Bébé d'êtreavec lui au cours de l'examen de son corps juste après son crime d'empoisonnement ${ }^{(54)}$.

Tout au long du roman, la narration du roman a été toujoursfaite à la "troisième personne du singulier" ${ }^{(55)}$ (Il-Elle et leurs formes toniques) pour désigner Bébé, François, Jacques, Félix, Jeanne... aussi avons-nous voulu braquer la lumière sur cette fréquence en citant les pages où figure la fréquence pour souligner l'idée que ce n'est pas une autobiographie de l'héroïne Bébé Donge. Si c'est "L'autobiographie" ${ }^{1(56)}$ personnelle de Simenon, elle doit être écrite à la première personne du singulier (Je) et écrite par la personne elle-même.

En ce qui concerne l'instance narrative, elle a été assurée par plusieurs voix. Au début, l'avocat Me Bonniface affirme que ni la jeune fille Bébé ni François n'avaient été bien préparés "au mariage." ${ }^{(57)}$ Vers la fin du roman, la voix narrative fait entendre François confessant: «Je me rends compte que la solitude et l'inaction dans lesquelles j'ai laissé une femme(Bébé), habituée à une vie plus brillante...» ${ }^{(58)}$ La solitude se développe avec le temps et prend de plus en plus de place avec le temps dans l'existence humaine.

\footnotetext{
${ }^{(51)}$ Ibid.p.88, cf. p.212, 213, 214, 215, 216,

${ }^{(52)}$ Ibid.p.23, cf.24, 25,26,

${ }^{(53)}$ Ibid.p.24.

${ }^{(54)}$ Ibid.p.33.

${ }^{(55)}$ Ibid.p.9, cf. p.10, 11, 12, 13, 14, 15, 16, 17, 18, 19, 20, 21, 22, 23, 24, 25, 26, 29, 30, 31, $32,33,34,35,36,37,38,39,40,41,42,43,44,45,46,47,48,49,50,51,52,55,56,57$, $59,61,62,63,64,65,66,67,68,69,70,72,74,75,76,77,78,79,80,81,82,84,85,86$, 87, 95, 98, 103, 104,105,106,107,110,etc

(56) LEJEUNE, Philippe : Le pacte autobiographique, Seuil, 1974, p.33

(57) SIMENON, Georges:Op.cit., p.13cf.p.16,44,74,82,86,87,89,91,92, $94,123,125,131,169,181,202,237,238$

${ }^{(58)}$ Ibid.p.220,
} 
Dr Wael Fouad Naguib

La preuve est que François n'a pas cessé ses aventures avec les femmes faciles ni avec madame Jalibert même après le mariage avec Bébé. Le mariage n'a donc remédié ni à sa solitude ni à celle de Bébé. La solitude a gagné le coup et a vaincu les êtres humains. Ce drame déchirant de l'âme humaine se renforçait par la marginalisation du partenaire et la recherche de l'assouvissement ailleurs. François était un coureur de filles de joie avant et après le mariage, Bébé a choisi Mimi Lambert comme remplaçant de François. Félix se plaisait lui aussi à des aventures en dehors du mariage, et Jeanne sa femme l'acceptait à condition de ne pas prendre place sous le toit de sa maison. Le père de Bébé et de Jeanne trahissait leur mère qui, à son tour, trouvait des attaches à l'extérieur du mariage mais sans trahison au sens propre du terme. Elle se détachait de lui.

Qui a donc raconté le roman ? Ce n'est pas la belle-sœur de François qui racontait le roman. La preuve est que 1':«On ne parlait d'elle comme on parle de quelqu'un d'autre, comme par exemple de sa sœur Jeanne.» ${ }^{(59)}$ Ce n'est pas non plus Félix le frère de François : «prononçait ces mots naturellement. On avait rencontré Jeanne Donge, simplement, une petite femme active, boulotte, toujours en mouvement, la femme de Félix Donge. Car les deux sœurs avaient épousé les deux frères.» ${ }^{(60)} \mathrm{Si}$ François avait narré le roman, il aurait utilisé le pronom personnel "Je" alors que la narration a été faite à la troisième personne du singulier (Il). Le même cas s'applique à l'héroïne principale dont le roman porte le prénom et le nom de famille de son mari. De même, ni la bonne Nicole ni la belle-mère de François n'ont rien raconté : "Nicole était un poison et les deux femmes (Mme d'Onnevilleet la bonne Nicole) se disputaient comme des filles du même âge, bien que la petite bonne n'eut que dix-neuf ans.» ${ }^{(61)}$

Pour la fonction de communication du narrateur omniscient avec le narrataire, nous citons : «N'arrive-t-il pas qu'un moucheron à peine visible agite davantage la surface d'une mare que la chute d'un gros caillou? Ainsi en fut-il ce dimanche-là à la Chataigneraie. D'autres dimanches, pour les Donge, sont restés en quelque sorte historiques, comme le dimanche de l'orage, quand le hêtre s'est

${ }^{(59)}$ Ibid.p. 15

${ }^{(60)}$ Ibid.pp.15-16

${ }^{(61)}$ Ibid.p.64 
Analyse stylistique de l'écriture de Georges Simenon dans La Vérité

abattu " trois minutes après le passage de maman ", ou encore le dimanche de la grande dispute, celle qui a brouillé les deux ménages pour plusieurs mois.» ${ }^{(62)}$

Pour la fonction narrative, dès les premières lignes de La vérité sur Bébé Donge, nous avons relevé un narrateur dont on ne sait pas le prénom. Il a toujours été désigné par le pronom indéfini "On". En fait, il ne fait pas partie de l'ensemble des personnages du roman, donc il est extra-diégétique et témoin du récit. La preuve est qu'au début du roman, nous relevons l'usage du pronom "On": «Ce dimanche-là, au contraire, qu'on pourrait appeler le dimanche du grand drame...» ${ }^{(63)}$ C'est le dimanche du crime perpétré par Bébé contre son mari François. En plus, le même narrateur est omniscient des autres grands événements de la vie du couple. De même, il a affirmé son omniscience de la vie du couple Bébé-François. En plus, dès la deuxième page du roman, il a donné plus de détails, sur les circonstances du crime de l'attentat à la vie de François. Il a citéla date du crime «On était le 20 aout.» ${ }^{(64)} 1$ De même, il a précisé les circonstances temporelles du jour même du crime : «Le soleil était déjà levé, le ciel d'un bleu lavé d'aquarelle, l'herbe humide et odorante.» ${ }^{(65)}$ Il accompagnait les divers personnages au cours de toutes leurs actions et réactions. A titres d'exemples, nous citons : «Dans la salle de bains, François se donna tout juste un coup de peigne, et il descendit en pyjama et en sandales, pénétra dans la cuisine où Clo, la cuisinière, à peine plus vêtue que lui, versait lentement l'eau bouillante dans la cafetière.» ${ }^{(66)}$ Il donne plus d'informations sur les habitudes du personnage principal du roman François: «Je ne peux pas préparer mes poulets bonne-femme sans champignons» ${ }^{(67)}$

\footnotetext{
${ }^{(62)}$ Ibid.p. 9 cf.p. $15,20,31$,

${ }^{(63)}$ Ibid. p9 cf.10, 11, 13, 14, 15, 16, 17, 18, 19, 20, 21, 22, 23, 25, 30, 31, 32, 46, 47, 49, $55,59,60,61,62,65,68,76,77,78,79,81,84,85,105,107,114,127,130,133,138$, $140,141,143,144,151,167,168,186,187,188,194,199,221,227,228,231,234,237$, $240,241$.

${ }^{(64)}$ Ibid.p.10, cf.11,18,

${ }^{(65)}$ Ibid.p. 10

${ }^{(66)}$ Ibid.p.10 cf.18,19,20,25,31,

${ }^{(67)}$ Ibid..p.13 , cf.p.14,15
} 
Dr Wael Fouad Naguib

Pour la fonction idéologique, le narrateur parle au lecteur à propos du bourg de la Chataigneraie : «...et on n'en(champignons) vend pas au bourg.» ${ }^{(68)}$ Il lui donne plus de précisions sur les distances : «Il n'y avait que quinze kilomètres pour la ville.» ${ }^{(69)}$ Sur les activités des gens du bourg le narrateur omniscient a dit: «Beaucoup de vélos sur la route. On les remarquait surtout dans la cote de BelAir parce que les cyclistes étaient obligés de marcher en poussant leur machine. Déjà des pique-niques en préparation à la lisière des bois.» ${ }^{(70)}$

Pour la fonction généralisante, nous avons relevé qu'il rapporte le point de vue du personnage masculin principal quand il juge le comportement de l'héroïne principale Bébé: «Maintenant encore, il (François) avait l'impression qu'elle (Bébé) avait entrouvert la bouche, comme quand on est sur le point de faire une remarque et qu'on se ravise en jugeant que cela n'en vaut pas la peine.» ${ }^{(71)} \mathrm{Le}$ narrateur omniscient ouvre la voie aux personnages importants pour dire eux-mêmes les événements dont ils étaient témoins comme Jeanne la sœur de Bébé : «Pendant dix-huit ans, son petit cerveau avait travaillé tout seul et, toute seule aussi, elle(Bébé) avait tenté d'effacer le vilain souvenir de la Grecque et de l'agent de police s'étreignant sordidement sur la table à repasser la lingerie. ${ }^{(72)}$ Il a délégué aussi à l'avocat Boniface de raconter aux lecteurs le passé de la vie de la mère de Bébé par le discours direct:«...Votre belle-mère est une Chartier, jepense ?...Figurez-vous que je l'ai connue quelque peu quand j'étais jeune...Elle avait un frère, Fernand, qui était lieutenant de cavalière à Saumur où j'avais un cousin. Ce cousin avait hérité......» ${ }^{(73)}$

Pour la fonction de régie, c'est le même narrateur omniscient qui fait le choix du moment de l'apparition des personnages sur scène et les modes de désignation de chacun d'eux. Ceci souligne l'importance du rôle de chacun d'eux par rapport aux autres. Nous

\footnotetext{
${ }^{((68))}$ Idem

${ }^{((69))}$ Idem

${ }^{((70))}$ Ibid.p.32

((71)) Ibid.p.32,

((72)) Ibid.p.181

${ }^{((73))}$ Ibid.p.195
} 
Analyse stylistique de l'écriture de Georges Simenon dans La Vérité avons relevé plusieurs procédés utilisés par lui. Dès les premières lignes du roman, Simenon commence à introduire le nom de famille du premier rôle masculin: «D'autres dimanches pour les Donge...» ${ }^{(74)}$ Quelques lignes après, avant de terminer la première page du roman, nous avons su que le premier personnage masculin est de la haute bourgeoisie de la campagne. Il est extrêmement riche, il a un seul frère, et il est marié. Puis à la fin, il a cité le prénom du premier rôle. Pour les rôles secondaires, le romancier cite le lieu où va apparaitre le personnage qu'il va présenter :«pénétra dans la cuisine où Clo, la cuisinière, à peine.... ${ }^{(75)}$ Quand il a voulu faire entrer le fils du couple sur scène, il a changé de décor, il a commencé par la tête de l'enfant, et a précisé sa relation par rapport à François sans nous dire son prénom : «François agita la main pour dire bonjour à son fils et l'enfant fit de même.» ${ }^{(76)}$ Pour préparer le lecteur à la présence de Bébé très bientôt sur la scène, il a repris une phrase de la première page du roman, puis: «Et on n'imagine pas ce que trois semaines,... Celui qui aurait annoncé que trois semaines plus tard Bébé Donge serait en prison...» ${ }^{(77)}$ Ensuite, le narrateur omniscient ajoute plus de détails sur elle «La femme la plus délicate, la plus jolie, la plus gracieuse...On ne parlait même pas d'elle comme on parle de......de sa sæur Jeanne.» ${ }^{(78)}$

Le narrateur omniscient a posé une question aux lecteurs pour se créer un "narrataire"(79)" extra-diégétique" ${ }^{(80)}$ au récit, pour se créer un "lecteur virtuel" ${ }^{(81)}$ ou pour faire croire aux lecteurs qu'il existe devant "le narrateur" un narrataire réel. En fait, après l'analyse, nous n'avons pas pu définir aucun trait caractéristique de ce narrataire extradiégétique. Ceci est au service d'élargir le lectorat de l'œuvre. De même, nous n'avons relevé aucun trait caractéristique du narrataire intra-diégétique. Simenon a commencé par une question posée dans le

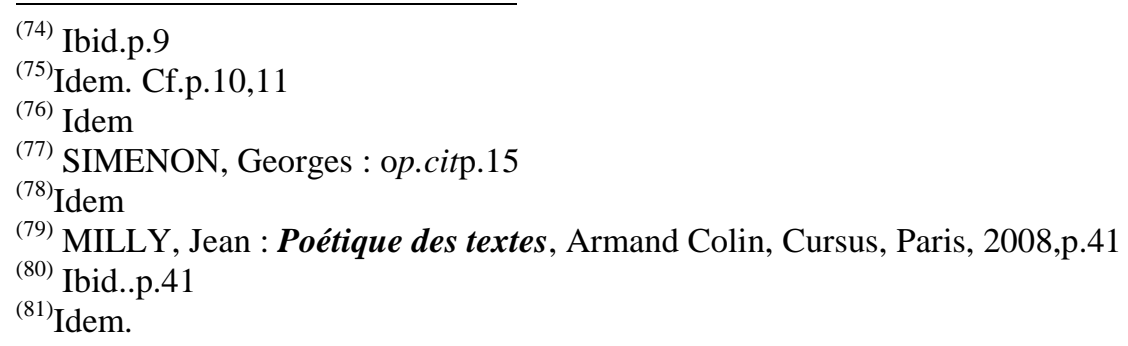


Dr Wael Fouad Naguib

but de créer ce lien avec le narrataire: «N'arrive-t-il pas qu'un moucheron à peine visible agite davantage la surface d'une mare que la chute d'un gros caillou ?» ${ }^{(82)}$

Dans la suite de l'analyse stylistique de l'écriture de Simenon dans La vérité sur Bébé Donge, il importe d'étudier la description des personnages du roman. Presque tous les personnages du roman sont la victime de la solitude et ils représentent les différents types de la société de la campagne française de l'époque, de la société française au cours de la première moitié du XX $X^{\mathrm{e}}$ siècle. Apparemment, les partenaires de chaque couple essayent de se convaincre de la réussite de leur mariage mais au fond ils partagent la souffrance de la solitude $^{(83)}$.

En gros, ni François ni Bébé ne garde aucune image positive du concept du mariage et de ses principes. Différemment, chacun d'eux prend l'autre pour un outil et un objet de plaisir et de satisfaction. Rien en eux ne comble le vide de l'existence. Selon Jeanne, dès son bas âge, Bébé n'avait pas "d'amies" ${ }^{(84)}$ et selon sa mère, François était une "fourmi de travail" (85).

De même, presque tous les personnages du roman souffrent de la solitude. Selon la psychologie, dans la majorité des cas, l'enfance pourrait contrôler nos choix dans le futur. Simenon a bien travaillé ses personnages et les sentiments qui les ont travaillés. Pour un critère objectif de classement des personnages nous avons vérifié l'œuvre même. Sur deux cents quarante-neuf pages, nous avons relevé cent pages sous la forme du "récit" ${ }^{(86)}$ alors que le reste des pages sont des

${ }^{(82)}$ Ibid.p. 15

(83) Idem

${ }^{(84)}$ Ibid.p. 175

${ }^{(85)}$ Ibid.p. 152

(86) Ibid.p.9 cf.p.10, 11, 14, 16, 29, 30, 31, 33, 34, 35, 39, 44, 45, 46, 49, 50, 55, 56, 57, 65, $67,68,69,74,77,78,79,80,81,84,86,87,95,97,98,101,103,104,107,111,115$, $116,121,124,125,127,138,139,141,142,142,148,151,152,153,157,159,160$, $162,181,183,184,186,187,188,189,190,193,194,195,217,219,221,222,223$, 230, 235, 241. 
Analyse stylistique de l'écriture de Georges Simenon dans La Vérité

"dialogues" (87) bien nets. Cetteclassification nous a permis de distinguer les personnages principaux de ceux qui ont été secondaires. En voici, les personnages principaux : Bébé, François, Jeanne qui est la sœur de Bébé, Lulu Jalibert qui est l'amoureuse de François, Mimi Lambert qui est l'amie de Bébé et l'avocat de Bébé Me Boniface.

Bébé a expliqué ses sentiments, François a affirmé son indifférence envers Bébé et comment il s'en est rendu compte. Lulu Jalibert a commenté la personnalité et elle a justifié le comportement de François envers Bébé. Jeanne a été le coffret noir du passé de Bébé. Mimi Lambert a été la dernière solution recherchée par Bébé avant de réaliser son attentat à la vie de François. Maitre Boniface a analysé le crime sur les différents plans et la raison pour laquelle François est devenu la victime alors qu'il était un criminel libéré. En fait, François a massacré Bébé sur le plan psychique alors qu'elle a essayé effectivement de le tuer.

Nous interprétons la dominance du discours ainsi. Simenon veut préparer les lecteurs à la question d'existentialisme traitée dans le roman, au discours qui sera lu pour bien suivre de près les personnages qui s'expriment «Trois semaines plus tard! On dit :Dans trois semaines... Ou bien...: - il y a trois semaines... ${ }^{(88)}$.Le romancier veut faire apprendre aux lecteurs comment l'héroïne dont le roman porte le prénom est vue par les uns et les autres dans son entourage ce qui capte l'attention du lectorat dès le début. Elle s'excelle à se montrer en cas de révolte. Plusieurs fois, elle s'est servie de plusieurs moyens entres autres ses habits. A titre d'exemples, nous citons : «...Quelle femme délicieuse!

(87) SIMENON, Georges :op.cit.p.10, cf.p.15, 16, 17, 18, 19, 20, 21, 22, 23, 24, 25, 26, 27, $31,32,33,35,36,37,38,39,40,41,42,43,44,45,47,48,49,50,51,52,53,54,55$, $58,59,60,61,62,63,64,65,66,67,69,70,71,72,73,75,76,77,78,79,81,82,83$, $84,85,86,87,88,89,90,91,92,93,94,95,96,97,98,99,100,101,102,103,104$, $105,106,107,108,109,111,112,113,114,115,116,117,118,119,120,121,122$, $123,124,125,126,128,129,130,131,132,133,134,135,136,137,138,139,140$, $141,142,143,144,145,146,147,149,150,151,154,155,156,157,158,159,160$, $161,162,163,164,165,166,167,168,169,170,171,172,173,174,175,176,177$, $178,179,180,181,182,183,185,187,189,190,191,193,195,196,197,198,199$, 200, 201, 202, 203, 204, 205, 206, 207, 208, 209, 210, 211, 212, 213, 214, 215, 216, $218,219,220,224,225,226,227,228,229,230,231,232,233,234,235,236,237$, 238, 239, 240, 241, 242, 243, 244, 245, 246, 247.

${ }^{(88)}$ Ibid.p. 15 
...Elle est plus séduisante que jamais...

Dr Wael Fouad Naguib

...Il n'y a personne pour s'habiller comme elle.... ${ }^{(89)}$

Le romancier veut faire parler des personnages importants par rapport aux autres pour faciliter la compréhension du drame. Bébé a reproché à son mari sa conduite provocante avec elle et avec les autres. Il aurait dû la traiter avec beaucoup de respect mais en fait : «...Ne me regarde pas avec cet air de te moquer du monde! lui répéter souvent sa femme, Bébé Donge. " ${ }^{(90)} \mathrm{La}$ phrase de Mme D'onneville qui est la mère de Bébé a été bien utilisée au service de la narration pour préparer le lectorat à imaginer un drame passionnel. En voici les paroles de Mme d'Onneville : «...Tu ne sais donc pas que ton mari et la belleMme Jalibert... Toute la ville est au courant...Certains le prétendent que Jalibert lui-même le sait et ferme les yeux...» ${ }^{(91)} \mathrm{Ce}$ fait a été très longtemps nié par Bébé au cours de l'interrogatoire après le crime : «...Je ne m'en suis pas inquiétée... Cela ne m'aurait pas affectée.» ${ }^{(92)}$ et par François lui-même vers la fin du roman. «Il n'y a pas d'affaire passionnelle....» ${ }^{(93)}$ De même, Jeanne la sœur de Bébé a ouvertement dit à sa mère:«...Mais non, maman, elle n'est pas folle... Il y a certainement des choses que nous ne savons pas..... Bébé a été toujours secrète...

....Elle n'a jamais eu de santé......

...Ce n'est pas une raison....Si tu ne l'avais pas tant gâtée» ${ }^{(94)}$

A l'inspecteur qui va ouvrir l'enquête avec elle, Bébé lui dit à propos de François : «...Vous serez mieux pour écrire dans le bureau de mon mari....Donnez-vous la peine de me suivre...» ${ }^{(95)}$ En ce tempslà, Bébé a déjà éprouvé le sentiment de délivrance de l'autorité de François, il n'existait plus pour elle alors qu'elle parle de lui en disant mon mari.

Lulu (Olga) Jalibert a décrit à François le caractère de Bébé : «...Le malheur pour toi, vois-tu, (François) c'est que ta femme ne soit

\footnotetext{
${ }^{(89)}$ Ibid.p.15 cf.p.16

${ }^{(90)}$ Ibid.p.13cf.p.12

(91) Ibid.p. 18

${ }^{(92)}$ Ibid.p.90

${ }^{(93)}$ Ibid.p.101,cf.p.66,93,208

(94) Ibid.pp.47-48

${ }^{(95)}$ Ibid.p.54
} 
Analyse stylistique de l'écriture de Georges Simenon dans La Vérité

pas une femme, mais une jeune fille...Hélas ! elle le restera toujours... Elle est incapable de te suivre... son rêve est de descendre toute sa vie une rivière au fil de l'eau, dans un cadre poétique, en murmurant des mots d'amour à l'homme ramant en face d'elle...» ${ }^{(96)}$ Cette idée du romantisme excessif de Bébé a été fréquente dans le texte du roman.

Jeanne a souligné à François l'importance de Mimi Lambert pour Bébé. François la détestait : «...Son amitié était, pour Bébé, plus qu'un soutien... Elle était parvenue à donner un sens à sa vie...Dans ces conditions, elle n'avait pas le droit, à cause d'une vexation, surtout causée par un homme, de l'abandonner à ellemême...Pourquoi souris-tu?» ${ }^{(97)}$ En plus, à propos de Bébé, elle a affirmé à François : «...Bébé a toujours vécu toute seule...» ${ }^{(98)}$ Devant l'avocat Boniface, Bébé a accusé à son tour François : «...Il ne $s$ 'inquiétait pas demoi.» ${ }^{(99)} 1$ A côté de lui(François) : «Je souffrais trop...» ${ }^{(100)}$ Longtemps avant l'amitié de Bébé avec Mimi Lambert, Bébé a essayé encore une fois de pousser François à modifier son comportement : «...Sais-tu à quoi je pensais, François ?... Que désormais nous vivrons toujours ensemble, que nous vieillirons, que nous mourrons ensemble.» ${ }^{(101)}$ Vers la fin du roman, Maitre Boniface a précisé la maladie de François : «...cruauté morale... Vous êtes atteint de cruauté morale, mon petit ami... Ha ! Ha !... Le tribunal vous condamne à vingt ans d'hopital... Sxur Adonie...emmenez le condamné !...» ${ }^{(102)}$ A l'hôpital tout juste après être sauvé de l'arsenic, François s'est laissé à un retour en arrière pour revoir ses dix ans de mariage avec Bébé et enfin, il s'est considéré «...Idiot !...Idiot !...Idiot !...» ${ }^{(103)} \mathrm{Au}$ fond, il n'a pu se libérer de ses attaches avec les filles de ses aventures amoureuses même après le mariage avec une femme aussi belle que Bébé : «...Il y a des années que je couche avec elle,

\footnotetext{
${ }^{(96)}$ Ibid.p.160,cf.p.135

${ }^{(97)}$ Ibid.p. 145

(98) Ibid.p. 180

${ }^{(99)}$ Ibid.p. 212

${ }^{(100)}$ Ibid.p.213

(101) Ibid.p. 121

(102) Ibid.p.225

${ }^{(103)}$ Ibid.p.156,cf.p.156,
} 
mais ce n'est pas ce qu'on appelle une maitresse...» ${ }^{\text {(104) }}$ Trop tard, après dix ans de mariage, François s'est rendu compte de sa fausse conduite avec la fille qu'il avait choisie pour épouse.

Tout juste après avoir été sauvé de l'arsenic, dans la salle de bain de sa maison, François n'a pas voulu suivre le docteur Jalibert qui a été obligé de faire appel au Palais de Justice de la Chataigneraie pour arrêter la criminelle Bébé. François a déclaré : «Je ne veux absolument pas, docteur.» ${ }^{(105)}$ sans avoir parlé avec Bébé, même sans savoir si elle l'a fait exprès, sans en être certain de "la vérité" ${ }^{(106)}$ de ce qui s'est passé. Bébé a demandé à François : "Promets-moi que, quoi qu'il puisse advenir, tu seras toujours sincère avec moi...Promets-moi de toujours me dire la vérité, même si cela devait me faire de la peine... Ce serait trop vilain de vivre toute sa vie l'un à côté de l'autre dans le mensonge...» ${ }^{(107)}$ Bébé avait longtemps souffert de sa vie sous le toit de son père qui avait eu des attaches en dehors du mariage et avait créé trop de mensonges. Il avait toujours trompé sa femme. Bébé insistait à ne pas répéter le même cercle vicieux. Mais, tout le long de dix ans de mariage, François n'avait pu découvrir la vérité de Bébé alors,selon lui dès la première rencontre, Bébé «l'a mis aussitôt à l'aise...» ${ }^{(108)}$ Elle a pu distinguer sa personnalité. L'angoisse de la solitude faisait souffrir non seulement Bébé mais aussi sa sœur Jeanne qui dit : «La vérité, c'est que je la connaissais à peine...» ${ }^{(109)}$ Vers la deuxième moitié du XIX ${ }^{\mathrm{e}}$ siècle Baudelaire a lancé l'idée d'aller vivre ailleurs pour trouver du nouveau, puis Rousseau a abordé le mal du siècle, J.Anouilh a initié ses lecteurs à l'Existentialisme, après lui, Simenon a fait de son mieux pour mettre à nu l'homme du début du XXème siècle rangé par l'angoisse de "la solitude"(110).

Quant à la description des personnages, Simenon excelle à varier les types des personnages qui appartiennent pour la majorité à la petite et à la haute bourgeoisie. L'objectif de cette description physique et psychique de ces deux héros principaux est de montrer

\footnotetext{
${ }^{(104)}$ Ibid.p.130

${ }^{(105)}$ Ibid.p.39

(106) Ibid.p.96, cf.p.100,104,122,174, 22,222,

(107) Ibid.p.22,

(108) Ibid.p.69,cf.p.181,

(109) Ibid.p. 174

${ }^{(110)}$ Ibid.p.187, cf.p.187,190,216,229,246
} 
Analyse stylistique de l'écriture de Georges Simenon dans La Vérité

d'un côté l'opposition des caractères de Bébé et de François et de l'autre côté l'attachement de François aux plaisirs sexuels.

A propos de Simenon, dans Larousse Encyclopédie, il a été écrit : "Ayant débuté à 16 ans comme journaliste à la Gazette de Liège (il y tient la rubrique des faits divers mais y donne déjà des contes, souvent érotiques)» ${ }^{(111)}$ En fait, selon l'autobiographie de Simenon sur Wikipédia: «Durant cette période, il approfondit sa connaissance du milieu de la nuit, des prostituées, de l'ivresse d'alcool, des garçonnières en ville» ${ }^{(112)}$. Tout le long du roman, pour la description physique des femmes, Simenon citait beaucoup de détails et de scènes de rapports intimes avec son épouse et les autres «de nombreuses aventures?...Assez nombreuses...La plupart du temps sans importance, souvent sans lendemain» ${ }^{(113)}$

Jusqu'à la fin du roman, les lecteurs assistent à l'évolution psychique de Bébé et de François pour aider à déchiffrer le "drame" ${ }^{114)}$ de point de vue de chacun des deux personnages principaux. Par intention, Simenon n'a pas mis fin à la vie de François pour convaincre tout homme de se rendre compte de la nécessité de changer pour le mieux. "Ce n'est plus le même homme depuis que Bébé....» ${ }^{(115)}$ En plus, Simenon n'a pas voulu encourager les femmes en ce temps-là à tuer leurs maris parce qu'il y a toujours une autre solution fiable pour découvrir la vérité del'héroïne dont le prénom constitue le titre du roman. Après l'attentat à la vie de son mari, au cours de l'interrogatoire, Bébé a répondu à la question de l'inspecteur«Question: En somme, vous aviez une sensation de délivrance ? Réponse : Oui.» ${ }^{(16)}$ Etant «Un être aérien, immatériel, sorti d'un recueil de poésies» ${ }^{(117)}$ Bébé s'était sentie délivrée d' «un homme pratique, sans imagination.» ${ }^{(118)}$ En fait, François était un riche industriel mais "pauvre"»" ${ }^{(119)}$ en émotions.

\footnotetext{
${ }^{(111)}$ https://www.larousse.fr/encyclopedie/personnage/Georges_Simenon

${ }^{(112)}$ https://fr.wikipedia.org/wiki/Georges_Simenon

${ }^{(113)}$ SIMENON, Georges: $\underline{O p . c i t . p p .93-94}$ cf.p. 31,41,57,75,107,116,130,135,161,181,186,

${ }^{(114)}$ Ibid. p. 9 cf.p. $40,93,141,197,215$,

(115) Ibid.p. 247

(116) Ibid.Op.cit.p.215,

${ }^{(117)}$ Ibid.Op.cit.p.16cff.p.30,31

${ }^{(118)}$ Ibid.Op.cit.p.34cf.p.31

${ }^{(119)}$ Ibid.Op.cit.p.245,cf.p.245,245,246
} 
Dr Wael Fouad Naguib

A la fin du roman, Bébé lui a conseillé de «demander le divorce et de refaire ta vie...Ce n'est pas parce qu'on a raté tous les deux ....» ${ }^{(120)}$ Ceci nous mène à l'analyse du "schéma actantiel"6 du roman La vérité sur Bébé Donge.D'habitude, il y a plusieurs schémas possibles mais nous avons choisi celui de l'hérö̈ne principale Bébé qui est plus compliqué et plus intéressant.

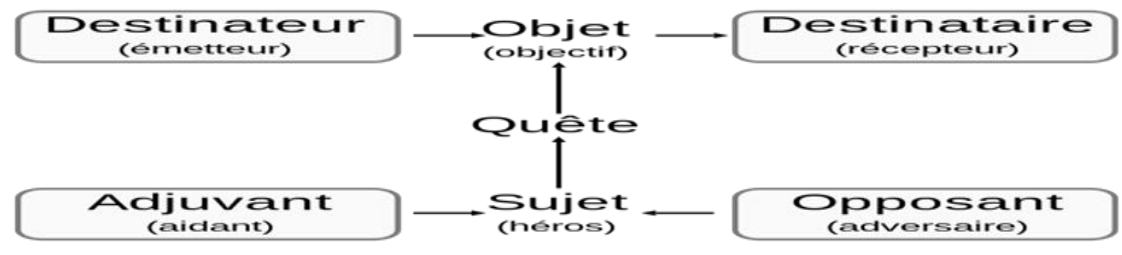

Le sujet(l'héroïne) c'est Bébé. Elle est en quête de a)se libérer de l'angoisse de la solitude b) de réaliser son bonheur personnel. Le destinateur c'est le Destin, alors que le destinataire c'est la Société avec toutes ses contraintes à l'époque de l'écriture du roman. Les opposants de Bébé sont assez nombreux parmi lesquels nous citons les plus importants : les parents de Bébé, l'époux François et ses parents, Lulu Jalibert qui représentent les filles de joie fréquentéesmême après le mariage par François, le travail de François, la bonne grecque et son amant. Pour les adjuvants, nous mentionnons les plus cités : le fils Jacques, Mimi Lambert, Jeanne la sœur de Bébé, les médecins, le substitut de Parquet, l'avocat Me Boniface, et le jury du tribunal.

En effet, les personnages neutres et/ou passifs qui regardent les événements sont beaucoup plus nombreux que les opposants qui dépassent à leur tour les adjuvants de Bébé. Nous soutenons l'idée que Simenon se montre aux lecteurs comme l'avocat et le protecteur de la femme et de ses problèmes relatifs à sonépoque. Simenon accorde une place centrale à la femme parce qu'il refuse de lui reléguer "le second plan" ${ }^{(122)}$

Passons à la mise en discours et l'étude des temps verbaux. En fait, l'imparfait du mode indicatif constitue le second plan par rapport au présent du mode indicatif qui occupe le premier plan ceci pour

\footnotetext{
${ }^{(120)}$ Ibid.Op.cit.p.244

(121) GREIMAS, Algirdas-Julien : Sémantique structurale, recherche et méthode, Larousse, 1966

${ }^{122)}$ NTSOBE,André-Marie : La vision de la femme chez Simenon et Senghor, Traces $\mathrm{n}^{\mathrm{o}} 16,2005, \mathrm{p} .227$
} 
Analyse stylistique de l'écriture de Georges Simenon dans La Vérité

mettre en relief la fausse conduite de François avec Bébé et la raison de la narration et de l'attentat à la vie de François. Ceci a pour but de rendre palpable la solitude dont souffre l'héroöne très tôt au début de son enfance, tout le long de sa jeunesse et enfin au cours de son mariage. "On n'a pas le droit de prendre un être, une jeune fille insouciante, sur la plage de Royan, de l'amener dans une maison et là, soudain, de l'abandonner à sa solitude. Pas même à sa solitude! A la solitude dans une atmosphère étrangère et qui peut paraitre hostile !» (123)

De même, l'emploi du présent de l'indicatif a pour rôle de poser la question primordiale illustrative du problème de Bébé et de François : « N'arrive-t-il pas qu'un moucheron à peine visible agite davantage la surface d'une mare que la chute d'un gros caillou ? ${ }^{(124)}$ L'image du moucheron revient sous la plume du romancier pour braquer la lumière sur la crise des partenaires ensemble et séparés. De même, le présent de l'indicatif fait circuler le problème de la race humaine et souligner aux lecteurs ou bien ou narrataires extradiégétiques le message «Les gens ne comprennent pas. Parce que s'ils comprenaient, il n'y aurait peut-être pas de vie possible ?» ${ }^{(125)}$ Philippe Beaussant a toujours dit dans Héloüse ${ }^{(126)}$ que la majorité des hommes ne changent pas de structures mentales malgré l'écoulement du temps c'est pourquoi ils apprennent peu de leurs actions et réactions passées. C'est une morale toujours en vigueur et d'ordre du jour pour chacun de nous. Cette idée est développée par de nombreux romanciers.

En plus, la majorité des temps verbaux utilisés au cours de la narration des événements, c'est-à-dire la diégèse (le récit, le second plan) se rapporte aux temps du monde raconté c'est-à-dire le passé simple et le plus que parfait de l'indicatif. L'emploi de ces temps varie d'un cas à l'autre : la description des décors, des états d'âmes, des comportements des différents actants à plusieurs moments illustratifs

\footnotetext{
(123) SIMENON, Georges : Op.cit.p.187

(124) Ibid.p.9,

(125) Ibid.p. 247

(126) BEAUSSANT, Philippe : $\underline{\text { Héloise }}$, Paris,Gallimard, NRF,1994.
} 
de crises, de leurs vies, ou bien au cours de leurs conflits nécessaires à la compréhension du texte.

À titre d'exemples de la description de l'intérieur des foyers : «Les chambres étaient claires. La maison était gaie. C'était vraiment la maison de campagne idéale.» ${ }^{(127)}$ poursouligner que l'angoisse de la solitude n'épargne ni les châteaux ni leurs habitants. La beauté interne des foyers n'est pas une solution à la vie solitaire ce qui a poussé Bébé à chercher une issue de son impasse. En effet, les parents de Bébé et de Jeanne sont devenus pauvres avant leur mariage avec François et Félix. Bébé enrichie représente le reste de la société mariée avec les riches seulement pour garder le train de vie.

Simenon s'adresse à un lectorat très diversifié intéressé à une cause humaine commune. Par l'emploi de l'imparfait de l'indicatif, Simenon résume brièvement la relation entre les personnages principaux même pendant leur relation intime au même endroit la rencontre hebdomadaire: "C'était le dimanche après-midi familial avec tout son vide somptueux et ses immenses champs de silence que chacun, enfoncé dans son fauteuil, traverse à son gré. Et celui qui ouvrait la bouche le premier semblait le premier arrivé d'un voyage sans histoire.» ${ }^{(128)}$ Cette même scène démontre que les deux partenaires doivent être sur la même longueur d'ondeau sein du mariage. C'est la solution fiable à l'angoisse de la solitude pour les mariés.

Pour la description des relations entre les personnages, voici : encore une fois l'imparfait de l'indicatif «François et Félix se ressemblaient comme des jumeaux ...Félix, comme son frère, avait le fameux nez des Donge. Même taille et corpulence.» ${ }^{(129)}$ Alors le type de relation entre François et Bébé est :«...se heurter ainsi l'un l'autre pendant...Dix ans !....» ${ }^{(130)}$ relève qu'il n'y avait aucune entente entre François et Bébé qui : «C'était bien le fils d'une femme dont il avait la grâce, la faiblesse, le repliement sur soi-même» ${ }^{(131)}$.Pour décrire la relation de François et Jacques «Il avait de son père, le nez long et de travers. C'était frappant. Rien qu'à cause de ce trait, François ne

${ }^{(127)}$ SIMENON, Georges:Op.cit.p.11, cf.p.45

(128) Ibid.p.35, cf.p. $174,180,181,185,187,216$

${ }^{(129)}$ Ibid.pp.18-19, cf.p.34

(130) Ibid.p.156, cf.p.212

${ }^{(131)}$ Ibid.p.151 

Analyse stylistique de l'écriture de Georges Simenon dans La Vérité
pouvait le renier. Pour le reste, l'enfant ressemblait à sa mère....» ${ }^{(132)}$ Jacques considérait gravement son père comme on considère un étranger. ${ }^{(133)}$ La solitude et la distance s'imposaient au sein du couple dès le début de leur mariage «A ce moment, ils se disaient encore vous. D'ailleurs, après dix ans de mariage, il leur arrivait souvent de se vouvoyer.» ${ }^{(134)}$ Leur relation continuait à être basée sur la haine qui s'est substituée à l'amourentre les deux partenaires plusieurs années avant le crime. ${ }^{(135)}$

A titres d'exemples de l'emploi de l'imparfait de l'indicatif entant qu'outil du romancier pour renforcer l'atmosphère d'engourdissement d'habitude, et de solitude des personnages principaux, nous citons : «C'étaient tous les dimanches à recommencer! François faisait le marché Samedi, entassait dans l'auto... Chacun lui envoyait sa liste,...Si sa femme ne dormait pas, elle ne faisait pas de bruit» ${ }^{(136)}$

En plus, nous relevons la monotonie des états d'âmes des personnages principaux pour de très longues durées mises en relief par l'emploi fréquent de l'imparfait : «Parce qu'il n'y avait là personne, à part son frère Félix,...» ${ }^{(137)}$ Par son expérience d'ancien amateur de filles de joie, la déconsidération de Bébé par François se rapportait à sa passivité au cours de la copule. «Il n'aimait pas sa peau trop blanche, ni sa façon passive...pendant l'amour» ${ }^{(138)}$ Cette relation corporelle et psychique trop intime devrait rendre leur lien conjugal trop fort mais la même relation vouait chacun d'eux de plus en plus à la solitude. Il est à remarquer que tous les personnages partageaient la même angoisse et l'assouvissement de leurs besoins chacun à sa propre manière. Les solutions appliquées par eux, l'excès de l'excès du romantisme(Bébé, Olga, Mme Flament, Mimi Lambert, la mère de

\footnotetext{
${ }^{(132)}$ Ibid.p. 11

(133) Ibid.p.151

(134) Ibid.p. 105

${ }^{(135)}$ Ibid.Op.cit.p. 210

(136) Ibid.Op.cit.p.13cf.p.9, 10, 11, 13, 25, 26, 31, 34, 35, 36, 44, 69, 75, 82, 98, 104, 105, $107,118,119,120,121$

${ }^{(137)}$ Ibid.Op.cit.p.34, cf.p.9, 18, 31, 82,

(138) Ibid.Op.cit.75,cf. p.78, 82, 105, 108, 115, 116, 119, 120, 121, 124, 125, 126, 127, 141, $143,144,145,147,148,149,151,152,153,154,155,157,158,159,160,162,163,168$ $170,174,178,180,181,183,185,186,187,188,189,191,204$
} 
Dr Wael Fouad Naguib

Bébé) ,l'excès du travail avec ou sans les pratiques sexuelles en dehors du mariage, ( Le docteur Jalibert, François, Félix, le père de Bébé). Après plus de quatre-vingt-ans, les mêmes problèmes persistent, les solutions proposées donnent preuves de leur inefficacité et le roman impose d'en trouver d'autres plus fiables comme il témoigne que l'âme humaine ne change pas de structures mentales, de réactions face aux mêmes problèmes.

Ceci confère plus de grandeur au romancier et à son roman dont l'écriture tend à se classer sous la rubrique d'école littéraire réaliste. Encore une fois, l'imparfait de l'indicatif soutient l'idée de cette tendance réaliste du romancier dans La Vérité sur Bébé Donge ${ }^{(139)}$. Parmi les exemples trop nombreux de ces descriptions réalistes de l'atmosphère, des rues, des constructions et des corps des personnages dans le roman, pour l'atmosphère choisie comme toile de fonddes évènements, nous citons: «On était le 20 aout. Le soleil était déjà levé, le ciel d'un bleu lavé d'aquarelle, l'herbe humide et odorante.» ${ }^{(140)} \mathrm{C}$ 'est l'atmosphère du jour même de la donnée de la mort à François par Bébé. Ce jour-ci, elle a choisi de se délivrer de l'autorité de conjugale de François. A la fin du roman, elle lui a dit : «Quand tu as bu le café...Tu n'existais déjà plus pour moi...» ${ }^{(141)}$

L'emploi de l'imparfait de l'indicatif pour cette description est très significatif parce que Bébé aurait pu mettre fin à la vie de François à partir du jour de la possession de l'arsenic au mois de mai. A la fin du roman, la veille et le jour même de l'annonce de la sentence du jury, il pleuvait et les larmes de François, de Jeanne, et de la bonne de Jacques coulaient parce qu'ils étaient tristes après l'enfermement de Bébé: «Et Marthe revenait du palais, toute mouillée, car elle avait oublié son parapluie dans la salle des témoins.» ${ }^{(142)}$ Cette pluie successive va de pair avec la fin tragique du roman, avec l'affection éprouvée de tout le monde pour Bébé, l'effondrement de François et sa demande d'être pardonné par Bébé et les pleurs du lectorat. Le roman débute avec la solitude de Bébé et se clôture avec sa claustration séparée dans une cellule et la claustration de François seul à la fois à la Chataigneraie et dans ses souvenirs avec

\footnotetext{
(139) -SIMENON, Georges: La vérité sur Bébé Donge, Paris, Gallimard,1940

(140) Ibid.p.10

(141) Ibid.p.10

${ }^{(142)}$ Ibid.p.236, cf.p.18, 22, 24, 29, 44, 47, 50, 238, 241, 242, 244.246 
Analyse stylistique de l'écriture de Georges Simenon dans La Vérité

Bébé. A la fin du roman, il a cessé d'être convaincu du caractère "orgueilleux"143) de Bébé et de sa famille. Bébé souffrait de l'absurdité de son "existence" ${ }^{(144)}$, l'un des termes et idées clés du roman. Son existence manquait de "vitalité" ${ }^{(145)}$ au cours de son mariage, la vie de Bébé était morne alors celle de François était pleine de vitalité. Dès le début du roman, nous relevons la fréquence du substantif de "la solitude" ${ }^{(146)}$ et ses dérivés.

Malgré la solitude qui rongeait la majorité des personnages du roman, la "jalousie" ${ }^{(147)}$ était le mobile qui contrôlait presque tout le temps leurs conduites. Aux yeux de François, la jalousie justifiait la conduite de Bébé avec lui. Il ne savait pas qu'elle était vouée à la solitude dès son bas âge selon les paroles de Jeanne. «Elle (Bébé)n'a pas eu de poupées...Elle n'a pas eu de petites amies» ${ }^{(148)}$ Selon l'avocat Me Boniface d'Emma (l'héroïne principale du roman) surnommé Bébé: «Les toilettes de votre femme, je ne dirai pas faisaient scandale, mais étonnaient, ainsi que son indifférence, si non son mépris, pour notre petite société.» ${ }^{(149)}$ Bébé avait un comportement cynique.

Dans La vérité sur Bébé Donge, Simenon analysait jusqu'au fond le problème d'ordre sexuel qui est majeur pour beaucoup de jeunes couples et l'un des conditions palpitantes de la réussite et de l'échec du mariage. "Car Bébé était incapable de faire l'amour !...» ${ }^{(150)}$ Sur le plan physique, il a décrit certains traits du corps de François qui ressemble à son propre corps. $\mathrm{Si}$ on compare la description de François : «François Donge n'était pas grand. Il était mince, mais dur, solide, avec des traits fins et ce long nez de travers si caractéristique, des yeux assez malicieux.» ${ }^{(151)} \mathrm{C}$ 'est une remarque sur le trait autobiographique de Simenon lui-même. Selon John Simenon,

\footnotetext{
(143) SIMENON, Georges: Op.cit.p.81

(144) Ibid.p.153, cf.p.156, 159, 174, 185, 197, 204, 209, 211, 212, 215, 238,

${ }^{(145)}$ Ibid.157, cf.p.158, 244, 245,

(146) Ibid.p.7, cf.p.58,115,120,144,152,157,162,181,216,217,218,229,246

(147) Ibid.p.89, cf.p.94, 119, 124, 143, 159, 160, 161, 171, 171, 208, 209, 210

(148) Ibid.p. 174 cf. 175

(149) Ibid.p.204

(150) Ibid.p.82,

(151) Ibid.p.13
} 
Dr Wael Fouad Naguib

«Les romans de mon père.... sont nourris de ses expériences, de ses observations.» ${ }^{(152)}$

Ce réalisme de "l'écriture"(153) 5 des sentiments s'appuie sur le présent de l'indicatif utilisé par l'auteur pour garder les idées qu'il défend encore palpitantes. En parlant à sa sœur Jeanne, Bébé a dit : «...Il y a longtemps qu'entre François et moi il n'y a plus rien de commun...» ${ }^{(154)}$ De même, le présent de l'indicatif va de pair avec la conduite corrompue de certains médecins voulant rendre services aux riches quand ils veulent acquitter l'un des leurs ayant commis vraiment un crime méritant de longues années de peine. Ils ont voulu plaider que Bébé était irresponsable de ses actes en désignant des experts capables de juger la criminelle irresponsable justifiée par des rapports sanitaires incorrects.

Lors de la rédaction de La vérité sur Bébé Donge, il y a presque quatre-vingt-ans, Simenon a fait dire à l'un de ses personnages: «En plaidant l'irresponsabilité, il (l'avocat Bonniface) est certain de son affaire et, de mon côté, je me charge des médecins qui seront désignés comme experts...» ${ }^{(155)} \mathrm{Ce}$ n'est pas le seul problème que Simenon relève à son temps mais c'est aussi un problème d'ordre du jour en 2019 «l'impréparation de la jeunesse d'aujourd'hui (1940) à la vie réelle et sur les contrecoups inévitables d'une éducation qui néglige systématiquement....» ${ }^{(156)}$ PourL. GAUBERT, Simenon c'est «L'état d'esprit d'un génie de l'écriture.» ${ }^{(157)}$

Nous ne pouvons pas terminer notre analyse sans passer à mettre en relief les divers emplois de la conjonction "Comme" dans des structures différentes. Leroman débute par l'emploi de la même conjonction en vue de marquer l'habitude, la monotonie et l'atmosphère étouffante menant au "grand drame." ${ }^{(158)} \mathrm{A}$ titre

\footnotetext{
${ }^{(152)}$ CARRON, Jérome: Interview de John Simenon, Point de vue, 3juillet2019 (pressreader)

${ }^{(153)}$ MILLY, Jean : Poétique des textes, Armand Colin,2008,p.17

(154) SIMENON, Georges: Op.cit.p.40

(155) Ibid.p.103,cf.p.67,102

(156) Ibid.pp.237-238

(157) GAUBERT, Jérome : “L'état d'esprit d'un génie d'esprit de l'écriture : Georges Simenon, https

:IIwww.Osez-écrire-votre-roman.com 21 février2018

${ }^{(15)}$ SIMENON, Georges : Op.citp.9, cf. p.9, 16, 61, 65, 68, 76, 78, 86, 98,104, 108, 144, $153,190,191,195$
} 
Analyse stylistique de l'écriture de Georges Simenon dans La Vérité

d'exemples de cet emploi, nous citons ce qui suit: "François s'éveilla vers six heures, comme chaque fois qu'il était à la campagne. ${ }^{(159)}$ Bébé tenait beaucoup à sa toilette et à son apparence : «Elle avait passé deux heures à sa toilette comme d'habitude.»(160)

La comparaison est une figure de style importante de l'écriture de Simenon. De même, le narrateur extra-diégiétique a utilisé le comparatif ou le "phore / l'outil comparatif "comme": «On ne parlait même pas d'elle comme on parle de quelqu'un d'autre, comme par exemple de sa sœur Jeanne» ${ }^{(161)}$. Le comparant c'est Bébé et le comparé c'est toute autre femme y inclus sa sœur Jeanne. Les deux sœurs étaient toutes les deux différentes chacune de l'autre et du reste des femmes de la Chataigneraie. Le but de cette comparaison est d'éclairer l'idée de la singularité de l'héroïne. La même idée est soutenue par la phrase suivante : «Il n'y a personne pour s'habiller comme elle.» ${ }^{(162)}$ parce qu'elle était «une femme délicieuse» ${ }^{(163)}$ et très "séduisante" ${ }^{(164)}$

Le but de la comparaison suivante est explicatif, «Il (Francois) $s$ 'était trompé comme les autres, ou plutôt il n'avait rien vu.» ${ }^{(165)} \mathrm{La}$ première du drame passionnel du couple héros du roman c'est que François ne s'était pas donné la peine de s'assurer si Bébé lui convenait comme épouse et mère de ses enfants. Il n'a pas pesé les conséquences des traits caractéristiques de la personnalité de chacun d'eux. Il s'est cru capable de la rendre heureuse et d'être heureux avec elle. Il n'a rien compris des avertissements précédant à l'attentat de sa vie. «Ici, encore, comme pour les morceaux de sucre, n'avait-il pas senti parfois comme un avertissement ?» ${ }^{(166)} 10$ Pour avancer une argumentation concernant le comportement aérien de la personnalité de Bébé, le narrateur extra-diégétique a cité la phrase suivante :«Bébé à l'arrière, laisser tremper les mains dans l'eau, comme sur une carte

\footnotetext{
${ }^{(159)}$ Ibid.p. 9

${ }^{(160)}$ Ibid.p. 17

${ }^{(161)}$ Ibid.p. 15

${ }^{(162)}$ Ibid.p.16

${ }^{(163)}$ Idem

${ }^{(164)}$ Idem

${ }^{(165)}$ SIMENON, Georges :Op.cit.p.37

${ }^{(166)}$ Ibid.
} 
Dr Wael Fouad Naguib

postale.» $^{(167)}$ L'outil de comparaison c'est la conjonction comme, la scène comparant est Bébé dans un bateau avec François et le comparé c'est la même scène dessinée sur une carte postale.

Le même but argumentatif de la comparaison est exprimé dans l'exemple suivant: «A vrai dire, le contact des lèvres devait lui apparaitre comme un rite peut-être nécessaire mais barbare.» ${ }^{(168)}$ L'outil comparatif c'est la conjonction "comme" le contact des lèvres c'est le comparant alors que l'acte lui-même qualifié de barbare et rite c'est le comparé. La fonction de la comparaison figurative descriptive en question c'est convaincre le lecteur que l'attentat à la vie de François était une conséquence prévue parce que la relation intime entre Bébé et François faisait souffrir atrocement Bébé. Elle se sentait obligée de faire l'amour avec son mari François, sans éprouver aucun plaisir sexuel, sans l'avoir voulu, sans jouir de cette rencontre. De même, malgré toute l'expérience de François avec les filles de joie, il n'a pas réussi avec Bébé. Quelques temps avant le crime, Bébé a proposé à François de se séparer d'elle puisqu'il ne menait pas une vie satisfaisante avec elle.

Après avoir été sauvé de l'empoisonnement, François a éprouvé le sentiment de la solitude et du vide interne «François se sentait vide comme il ne l'avait jamais été, vide et propre à la façon d'un animal que le boucher vient de débarrasser de ses entrailles......» ${ }^{(169)}$ Simenon prépare le lecteur à l'analyse psychique et mentale que François va entreprendre au sujet de sa relation avec Bébé. François a essayé d'explorer les raisons qu'il ignorait jusque-là. Selon le procès-verbal, Bébé a mentionné que la vraie raison de la faillite de ce couple c'est l'incommunicabilité entre les deux partenaires de caractères trop différents. Bébé était toujours de caractère aérien et sentimentale mais son corps «était parcouru comme d'un tressaillement de douleur» ${ }^{(170)}$ au cours de la relation conjugale. Dans cet exemple, l'outil comparatif c'est la conjonction "comme". Le but de cette comparaison c'est de décrire aux lecteurs

\footnotetext{
(167) Ibid.p.106

${ }^{(168)}$ Ibid.p.107cf.p.15, 18, 31, 37, 43, 44, 46, 49, 55, 56, 64, 68, 74, 81, 83, 94, 98, 100, 106, $107,119,121,122,127,130,130,134,139,141,144,145,151,152,153,154,157,158$, $159,160,163,168,171,175,178,179,180,188$,

${ }^{(169)}$ Ibid.p. 55

${ }^{(170)}$ Ibid.p. 107 
Analyse stylistique de l'écriture de Georges Simenon dans La Vérité

féminins surtout combien sa douleur est pénible. La relation corporelle intime avec son mari ressemblait pour elle à un ensemble de secousses douloureux sans aucune joie.

En fait, selon le comportement de Bébé au long du roman et les soins qu'elle accordé à son fils unique Jacques démontraient qu'elle s'attachait effectivement à son fils en tant que mère c'est pourquoi elle a préféré de se débarrasser de son mari parce que l'enfant a plus besoin d'une mère que d'un père. Au début de son mariage raté, Bébé a proposé à François de donner naissance à un enfant.

Le but de la comparaison suivante est de décrire la froideur entre elle et son mari : «Il (François) ne s'attendait pas à cette demande, surtout présentée avec sang-froid, presque comme une affaire.» ${ }^{(171)}$ Bébé a provoqué François par la manière de sa demande d'avoir un enfant, dès le début de leur mariage. Ceci a créé un grand fossé entre eux. Dans cet exemple le comparant c'est avoir un enfant alors que le comparé c'est une affaire. Pour un homme matérialiste comme François, nous croyons qu'il déconsidérait Bébé après cette discussion soulignant la froideur de leur relation dès leur début.

En fait, la figure de style "la répétition'” 2 sous ses différentes formes a attiré notre attention au cours de la lecture du roman. Nous avons choisi quelques-unes de toutes ces formes telles que "l'anaphore".3 Une anaphore est la répétition d'un mot, d'un nom ou de plusieurs mots en début de phrase. Elle permet de mettre l'emphase sur une idée, un objet, une personne. L'exemple ci-dessous met en relief la relation bien fondée entre François et son frère Félix tout le long de leur vie. "Tout était commun entre les deux frères. Ensemble ils travaillaient. Ensemble ils avaient monté les affaires que dans la ville on appelait les affaires Donge. Ensemble ils s'étaient mariés et ils avaient épousé les deux sœurs. Ensemble enfin, et à fonds communs,

\footnotetext{
${ }^{(171)}$ Ibid. p.149 cf.p.151, 152, 153，154, 157, 158, 159, 160, 163，171，178, 179 nb.La conjonction a été utilisée dans d'autres phrases non dans le but de comparaison ex.p.18, $31,32,42,48,52,75,87,93,107,118,120,130,135,137,139,146,147,148,149,158$, $171,176,186$,

2-https://narrationetcafeine.fr/figures-de-style-de-répétition/

3- https://www.laculturegenerale.com/anaphore-définition-exemples
}

Faculty Of Arts Journal 
Dr Wael Fouad Naguib

ils avaient transformé la Cha...,"172 Pour exprimer le dégout éprouvé par François envers sa femme Eugénie surnommée“'Bébé", ${ }^{173}$,le narrateur omniscient a cité ' Il n'aimait pas sa chair. Il n'aimait pas sa peau trop blanche, ni sa façon passive de s'abandonner, de garder les yeux ouverts et les prunelles sereines pendant l'amour.',174

Dans l'exemple suivant, nous allons analyser l'expressivité de l'emploi de "l'épiphore',175. "Une femme incapable de faire l'amour !.... Car Bébé était incapable de faire l'amour." 176 François était un amateur de femmes avant le mariage et il ne cessait pas de s'aventurer avec les filles de joie après le mariage et il racontait ses aventures à Bébé quand elle le lui demandait.

" 'L'anadiplose",177 consiste à réutiliser un ou plusieurs mots d'une phrase précédente comme point d'appui pour développer la phrase qui suit. François a reconnu son crime envers sa femme Bébé ' On n'a pas le droit de prendre un être, une jeune fille insouciante, sur la plage de Royan, de l'amener dans une maison et là, soudain, de l'abandonner à sa solitude. Pas même à sa solitude ! A la solitude dans une atmosphère étrangère et qui peut lui paraitre hostile !',178 L'angoisse de la solitude menaçait tout le monde et le menacerait.

A titre d'exemples de 1'“épanadiplose,"179, “Un couple marchait devant eux, un couple qui avait déjà l'air d'un couple.',180 Ni Bébé ni François n'ont eu le courage de vérifier s'il y a eu uneentente de personnalités entre eux. Elle lemettait à l'aise et lui se plaisait. Ils se sont vite mariés sans s'assurer de leur entente réciproque. 'Bébé ne l'avait pas fait par hasard. Dès leur rencontre, au bar du casino, elle avait agi en pleine connaissance de cause, il en était persuadé.', 181

\footnotetext{
172 - SIMENON,Georges:op.cit.p65, cf.p.74,120,148, 156,217,245

173 - SIMENON,Georges: op.cit. p.13,cf.p.23,43,43,44,45,47,50,51,

174 - Ibid.p.75,cf.p.120,127,141,160,187,247

175 - https://fr.wikipedia.org/wiki/épiphore

176 - SIMENON,Georges:op.cit.p.82 cf.p.129,135,212,244

177 - https://www.laculturegenerale.com/anadiplose-definition-exemples/

178 - SIMENON, Georges: op.cit. p.187,

179 - http://acceslitteraire.e-monsite.com/pages/stylistique/figures-deconstruction/epanadiplose.

180 - SIMENON, Georges:op.cit.p.74, cf.107

${ }^{181}$ - Ibid.p. 147
} 
Analyse stylistique de l'écriture de Georges Simenon dans La Vérité

"L'épizeuxe"182 consiste à répéter un même mot au moins une fois sans aucune variation dans une même phrase. " .....ma pauvre Jeanne, je suis un monstre.....Je suis un imbécile....Je suis un idiot, un pauvre idiot.....C'est moi qui suis responsable de tout !..." ${ }^{183}$ François s'est rendu compte que l'argent ne le rendait pas heureux avec Bébé qui n'a pas pu payer la dote. Bébé était plus riche en sentiments par rapport à son mari qui était riche en argent mais pauvre en sentiments.

Enfin, "le polyptote consiste à répéter un mot avec une variante" ${ }^{\prime 184}$ Le narrateur omniscient a voulu souligner l'un des traits caractéristiques de la personnalité de François: “'Il (François) n'aimait pas être dérangé par les autres et il ne les dérangeait pas volontiers." $" 185$

En guise de conclusion, nous avons fait état de quelques traits caractéristiques de l'écriture de la fiction de Simenon dans son œuvre. Nous ne prétendons pas tout dire sur l'analyse stylistique d'écriture mais nous avons essayé de relever les plus pertinents soit au niveau du récit soit au niveau du discours. En tant que héros, François et Bébé forment un couple présentatif les hommes de l'époque des années 1930-1940 comme ceux de notre temps contemporain. L'incommunicabilité entre le jeune couple et la solitude dont souffre chacun d'eux sont de nos problèmes d'ordre du jour. Si François cherche à remédier à sa solitude au sein du couple par des attaches en dehors du mariage, Bébé a choisi de s'enfuir de sa solitude en se créant un lien d'amitié avec une autre femme qui a eu une apparence physique et vestimentaire semblable aux hommes pour remplacer son mari indifférent à son égard et froid à ses sentiments conjugaux. La solitude a crevé les héros de Simenon et elle n'a pas cessé de dévorer les hommes qui sont appelés à apprendre à bien choisir leurs femmes et à bien communiquer avec elles afin de ne pas imposer à l'épouse de faire le choix entre le suicide et l'attentat à la vie du mari. Avons-nous

\footnotetext{
182 - https://www.wikiwand.com/fr/Epizeuxe

183 - SIMENON, Georges: op.cit.p.165

${ }^{184}$ https://narrationetcafeine.fr/figures-de-style-de-repetition/

${ }^{185}$ SIMENON, Georges: La vérité sur Bébé Donge, Paris, Gallimard, 1940,p.36

cf.p.67,68,101,102,108,121,122,

$123,129,133,137,145,161,171,184,186,190,192,196,202,215,228$.
}

Faculty Of Arts Journal 
Dr Wael Fouad Naguib

d'autres choix pour échapper à la solitude et à l'incommunicabilité ? Euvrons pour aider toute Bébé à mener une meilleure vie. En fait, Bébé a été le surnom de l'héroïne principale dont le vrai nom c'est "Eugénie" ${ }^{(186)}$ recouvrant toutes les filles. 13-5-2020

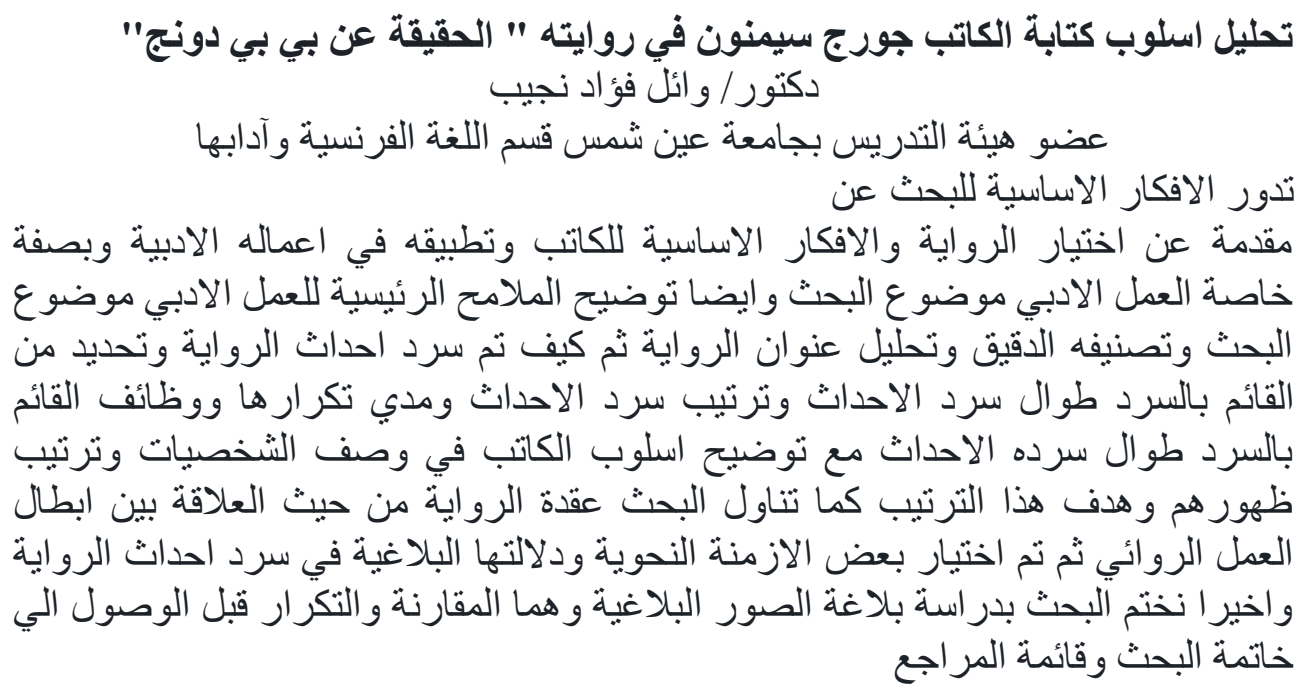

\section{Références}

Euvre de Georges SIMENON,

SIMENON, Georges : La vérité sur Bébé Donge, Paris, Gallimard, 1940

Euvrages critiques sur Georges Simenon:

LEMOINE, Michel: Ecrire l'homme, Paris, Gallimard, 2003,

LEURRIN, Michel et SCHWERIGHAEUSER,Jean Paul: Le Guide du polar, Histoire du roman policier français, Paris, Syros, "Les Guides culturels", 1987.

DE FALLOIS, Bernard: Simenon, Paris, Gallimard, col."Bibliothèque Idéale", 1961,p.16

CARLY, Michel : Simenon, les années secrètes : Vendée 1940-1945 , Le ChâteauD'Olonne : Ed.d'Orbestier, 2005

Articles critiques :

NTSOBE, André Marie: La vision de la femme chez Simenon et Senghor, Traces, n 16, 2005 ,

Articles critiques (source internet)

www.Dico-citations, Banks, RUSSELLE, La Réserve(2008)

La Chataigneraie :https://fr.wikipedia.org/wiki/Wikip.Accueil_principal

GAUBERT, Laure : L'état d'esprit d'un génie de l'écriture :Georges Simenon : www.Osezecrire-votre-roman 21 février 2018

${ }^{(186)}$ Ibid.p. 43 cf.p. 30 
Analyse stylistique de l'écriture de Georges Simenon dans La Vérité

SIMENON,Georges:https://fr.wikipedia.org/wiki/https//www.larousse.fr/encyclopedie/pers onnage/Georges_Simenon.Interview sur internet :

CARRON, Jérôme : Interview de John Simenon, Point de vue, 3juillet 2019

Euvres méthodiques consultées

BUFART-MORET, B. : Introdcution à l'analyse stylistique, Paris, Dunod.

DURRENMATT ,J. Stylistique de la poésie, Paris, Belin, 2005FORMILHAGUE, Catherine, SCANCIER-CHATEAU, Anne, Introduction à l'analyse stylistique, Paris, Bordas, 1991

MILLY, Jean : Poétique des textes, Paris, Nathan,1992,p.41

FONTANIER,Pierre, Les figures du discours , 1ère éd.1830, nouvelle édition,Flamarion, 1968.

FORMILHAGUE, Catherine, Les figures de style, Nathan-Université , coll. « 128 », 1995.

BOURNEUF, Rolland, et OUELLET Réal, l'univers du roman, PUF,1972.

GREIMAS, Algirdas-Julien : Sémantique structurale, recherche et méthode, Larousse, 1966

FONTAANIER, Pierre : Les figures du discours,Paris,DUNOD,2002

MOLINE, Georges : Eléments de stylistique française, Paris,PUF, 1986

Sites d'internet consultés pour la figure de répétition

Références des sites https://narrationetcafeine.fr/figures-de-style-de-répétition/

https://www.laculturegenerale.com/anaphore-definition-exemples

- https://fr.wikipedia.org/wiki/épiphore

http://acceslitteraire.e-monsite.com/pages/stylistique/figures-de-construction/épanadiplose 